\title{
MORE GROUPS THAT ARE JUST ABOUT FREE
}

\author{
BY GILBERT BAUMSLAG ${ }^{1}$
}

\author{
Communicated by M. Suzuki, January 31, 1968
}

1. A residually nilpotent group $G$ is termed parafree of rank $r$ if $G / \gamma_{i} G \cong F / \gamma_{i} F$ for $i=1,2, \cdots$, where $F$ is a free group of rank $r$ and $\gamma_{i} X$ is the $i$ th term of the lower central series of the group $X$. The object of this announcement is to make known the existence of certain nonfree parafree groups with the most extraordinary properties. Unlike the groups in [1] and [2] these groups are not finitely generated, but they have even more surprising properties.

THEOREM. Let $r>1$ be an integer and let $V_{1}, V_{2}, \ldots$ be any countable family of varieties, none of which is the variety of all groups. Then there exists a parafree group $G$ of rank $r$ such that

(i) $G$ is locally free;

(ii) $G / V_{i}(G) \cong F / V_{i}(F)(i=1,2, \cdots)$ (where $V_{i}(X)$ is the least normal subgroup of the group $X$ with factor group $X / V_{i}(X)$ in the variety $V_{i}$ );

(iii) $G$ is not free.

2. At present it is not known whether there are more than a countably infinite number of varieties. Thus we may take

$$
V_{1}, V_{2}, \cdots
$$

to be the set of all known varieties of groups, excluding the variety of all groups. So this set will contain for each finite group the variety generated by it, and also the variety $\boldsymbol{S}_{\boldsymbol{i}}$ generated by all soluble groups of derived length at most $i$. The resultant locally free, parafree group $G$ provided by the theorem will therefore have, in particular, the following properties:

(a) $G$ has precisely the same number of subgroups of finite index $j$ as the free group $F$ of $\operatorname{rank} r$ for $j=1,2, \ldots$;

(b) every finite homomorphic image of $G$ can be generated by $k$ elements;

(c) $G / \delta_{i} G \cong F / \delta_{i} F$ for $i=1,2, \cdots$ (where $\delta_{i} X$ is the $i$ th derived group of $X$ ).

3. The groups $G$ of the theorem are constructed as a colimit (i.e. direct limit) of free groups of rank $r$ in a very simple way.

\footnotetext{
${ }^{1}$ Support from the National Science Foundation is gratefully acknowledged.
} 
Let $G_{i}$ be the free group of rank $r$ freely generated by

$$
a_{i 1}, a_{i 2}, \cdots, a_{i r}
$$

for $i=1,2, \cdots$. For each $i \geqq 2$ put

$$
W_{i}=V_{1} \cup V_{2} \cup \ldots \cup V_{i} \cup S_{i}
$$

and choose

$$
u_{i} \in W_{i}\left(G_{i}\right)
$$

in such a way that

$$
a_{i 1} u_{i}, a_{i 2}, \cdots, a_{i r}
$$

generate a proper subgroup $F_{i}$ of $G_{i}$. Notice that such a choice is always possible and that $F_{i}$ is free of rank $r$ since $u_{i}$ lies in the derived group of $G_{i}$. Now let $\mu_{i}$ be the monomorphism of $G_{i}$ into $G_{i+1}$ defined by

$$
\mu_{i}: a_{i 1} \rightarrow a_{i+1,1} u_{i+1}, a_{i, 2} \rightarrow a_{i+1,2}, \cdots, a_{i, r} \rightarrow a_{i+1, r}
$$

This system of groups $G_{i}$ and monomorphisms $\mu_{i}$ determines a colimit $G$ of the groups $G_{i}$. Indeed we may simply think of $G$ as an ascending union of its subgroups $G_{i}$ where

$$
a_{i 1}=a_{i+1,1} u_{i+1}, a_{i j}=a_{i+1, j} \quad(i=1,2, \cdots, j=2,3, \cdots, r) .
$$

$G$ is then the desired group. We have still to show that $G$ has the right properties.

First we observe that $G$ is locally free since it is an ascending union of free groups.

Second we consider $V_{n}(G)$. Clearly

$$
V_{n}(G)=\bigcup_{i \geq n} V_{n}\left(G_{i}\right)
$$

Since $u_{i} \in V_{n}\left(G_{i}\right)$ for each $i \geqq n$,

$$
G_{i} / V_{n}\left(G_{i}\right)=G_{i-1} V_{n}\left(G_{i}\right) / V_{n}\left(G_{i}\right) \text {. }
$$

Therefore modulo $V_{n}\left(G_{i}\right), u_{i-1,1}, \cdots, u_{i-1, r}$, freely generate $G_{i-1} V_{n}\left(G_{i}\right) / V_{n}\left(G_{i}\right)$. Consequently

$$
V_{n}\left(G_{i}\right) \cap G_{i-1}=V_{n}\left(G_{i-1}\right) \text {. }
$$

Since this equality holds for all $i \geqq n$ we find that

$$
G / V_{n}(G) \cong G_{n} / V_{n}\left(G_{n}\right) \text {. }
$$

This is the required isomorphism. 
Thirdly it follows easily from these observations and the residual nilpotence of free groups that $G$ is parafree of rank $r$.

Finally we observe that $G$ is not finitely generated, but that $G / \gamma_{2} G$ is free abelian of rank two. Hence $G$ is not free.

\title{
REFERENCES
}

1. G. Baumslag, Groups with the same lower central sequence as a relatively free group. I: The groups, Trans. Amer. Math. Soc. 129 (1967), 308-321.

2. - Some groups that are just about free, Bull. Amer. Math. Soc. 73 (1967), 621-622.

Graduate Center, The City University of New York

\section{SOLVABLE AND NILPOTENT SUBALGEBRAS OF LIE ALGEBRAS}

\author{
BY DAVID J. WINTER ${ }^{1}$ \\ Communicated by G. B. Seligman, February 21, 1968
}

1. Introduction. We describe here some results on the structure of a Lie algebra in terms of its nilpotent and solvable subalgebras. Proofs will appear elsewhere.

In the following discussions, $F$ is an arbitrary field, $\mathscr{L}$ is a (finite dimensional) Lie algebra over $F$ and $V$ is a (finite dimensional) vector space over $F$.

2. Arbitrary Lie algebras. Let $\Re$ be a set of linear transformations in $V$ such that the Lie algebra generated by $\Re$ over $F$ is nilpotent. Then, as is well known, $V$ has a unique vector space decomposition $V=V_{0}(\Re)+V_{*}(\Re)$ (direct) where $V_{*}(\Re)$ is $\Re$-stable, $V_{0}(\Re)$ is $\Re$-stable and $\left.\Re\right|_{V_{0}(\Re)}$ consists of nilpotent transformations, and where $V_{0}(\Im)$ is maximal with respect to the latter two properties.

One has the following theorem, in spite of the fact that a nilpotent linear Lie algebra cannot always be triangulized over the algebraic closure of its base field.

TheOREM $1 .{ }^{2}$ If $F$ is infinite and $\Re$ is a subspace of $\operatorname{Hom}_{F}(V, V)$ such that the Lie algebra generated by $\mathfrak{N}$ is nilpotent, then there exists $N$ in $\Re$ such that $V_{0}(N)=V_{0}(\Re)$.

1 This research was done while the author was a National Science Foundation Postdoctoral Research Fellow at the University of Bonn.

${ }^{2}$ This is essentially Lemma 3.5 of [4, pp. 87-88]. The author has heard that an independent forthcoming paper of $\mathrm{R}$. Block contains material close to this theorem. 